\title{
Quantum Optics with Quantum Dots
}

\author{
A. Imamoḡlu \\ Departments of Electrical $\&$ Computer Engineering and Physics \\ University of Califonia, Santa Barbara, CA 93106
}

\begin{abstract}
Semiconductor quantum dots have emerged as promising candidates for studying quantum optical phenomena. Observation of photon antibunching and spontaneous emission limited coherence decay rates have demonstrated that quantum dots behave as artificial atoms. Cavity-quantum electrodynamics (QED) phenomena can be investigated using a single quantum dot embedded inside a photonic nanostructure, where both carriers and photons are confined within sub-micron length scales in all three dimensions. Since quantum dot location inside the cavity is fixed by the growth, this system is free of the stringent trapping requirements that limit its atomic counterpart. Possibility of fabricating photonic nanostructures with ultra-small optical-mode volumes and long photon lifetimes enhances the prospects for applications in quantum information processing.
\end{abstract}

\section{Introduction}

Quantum dots (QD) are semiconductor structures which provide full three-dimensional confinement for conduction band electrons and/or valence band holes [1]. There are a variety of quantum dot structures grown or fabricated using different techniques, resulting in different confinement length scales ranging from 2 to 100 nanometers. In most QD systems, a discrete optical excitation spectrum is observable only at cryogenic temperatures.

Here, we present quantum optics experiments carried out using self-assembled InAs QDs grown by Molecular Beam Epitaxy (MBE) [2]. Self-assembled InAs islands form spontaneously on GaAs surface during MBE growth: due to the lattice mismatch between the two semiconductors, the total film energy, including strain energy, interfacial energy and surface energy, is minimized through the formation of coherently strained islands. Formation of actual QDs is achieved by covering the smaller band gap material of the InAs islands with the wider band gap GaAs epitaxial film. Since InAs/GaAs systems has a type-I interface, both electron and hole levels are confined to the lower-bandgap (InAs) material, leading to a discrete optical excitation spectra $[2]$.

The exact shape, size and composition of the QDs are not known, making it practically impossible to make accurate theoretical predictions about the QD energy eigenstates. We work with samples with a random in-plane distribution of QDs, with a density ranging from $1 \times 10^{6}$ to $1 \times 10^{1} 1 \mathrm{QDs} / \mathrm{cm}^{2}$. The emission from the lowest energy electron-hole pair, referred to as the fundamental exciton transition, occurs in the wavelength range of $920-980 \mathrm{~nm}$ [2].

Several features differentiate optical signatures of QDs from that of atoms. First and foremost, a given single QD is naturally trapped in the GaAs lattice in which it is embedded: this feature makes it possible to carry out experiments on the same QD without having to worry about random thermal motion. This feature has already been utilized in experiments demonstrating single-photon source operation $[3,4,5]$ 
and simultaneous presence of bunching and antibunching in cascaded emission $[6,7]$. A second important feature is the fact that the oscillator strength of the fundamental exciton transition could range between 10 and 300: this is a collective enhancement of the optical transition strength arising from the fact that many atoms contribute to a single optical excitation [8]. A third and as yet unexplored feature of QDs is the completely different nature of hyperfine coupling arising from the fact that electrons in a QD are extended objects interacting weakly with many nuclei. Finally, the GaAs material in which InAs QDs are embedded, can be processed into a photonic nanostructure exhibiting high quality (Q) factor cavity modes; since modern semiconductor nanofabrication techniques allows the formation of sub-micron GaAs structures with very smooth surfaces [9]. Given the large index-of-refraction of semiconductors in general and GaAs in particular $\left(n_{r} \sim 3.5\right)$, such structures can provide full three-dimensional confinement for optical modes, with mode volumes approaching the fundamental limit of $\left(\lambda / 2 n_{r}\right)^{3}$, where $\lambda$ is the wavelength of light emitted at the fundamental electron-hole (exciton) transition.

Figure 1 shows a typical single QD photoluminescence (PL) spectrum. Here, a laser is used to create free electron-hole pairs in the the GaAs region surrounding the QD. These free carriers are then captured into the low energy discrete states of the QD and relax down to the lowest energy available electron and hole states in less than 100 psec [9]. Radiative recombination of the captured electron-hole pairs results in the pump-power dependent luminescence spectra depicted in Fig. 1.

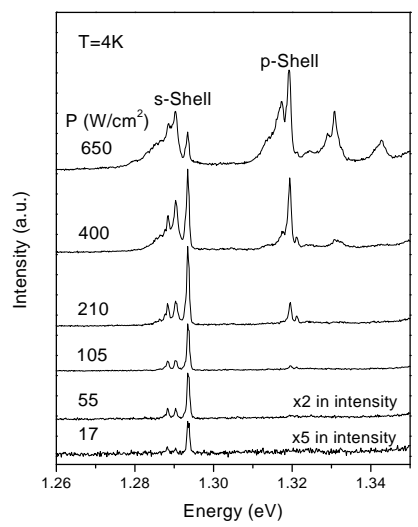

Figure 1: Photoluminescence spectra of a single quantum dot at various pump powers.

As can be seen in Fig. 1, the PL spectrum of a single QD is extremely rich. This richness arises (once again) primarily from the fact that the electron confinement length-scale is much larger than the lattice spacing, giving rise to multi-electron-hole excitations that differ in energy from the fundamental exciton emission by only a few meV. The best known example of the multi-exciton transitions is the biexciton line which appears when a photon is emitted from a QD containing two electrons and two holes, all in their ground motional states: the biexciton photon energy is red-shifted by about $3.5 \mathrm{meV}$ in most InAs QDs due to Coulomb correlation effects [6]. For a weakly excited QD, the resolution-limited single-exciton (1X) line dominates the spectrum (Fig. 1); increasing the excitation power results in the appearance of additional lines, 
most notably the biexciton line. Under non-resonant excitation, achieved by creating free electron-hole pairs in GaAs, the number of electrons captured by the QD need not equal that of holes: in this case, charged exciton lines with small red or blue detuned emission energies may also appear in the spectrum. Finally, under strong excitation, the QD spectrum develops a strong background and is qualitatively different from the spectrum of a single atom.

\section{Photon antibunching}

How can we be sure that the observed PL spectrum depicted in Fig. 1 originates from a single QD? The presence of resolution-limited sharp emission peaks proves that the observed system has full three dimensional confinement, and the large variation in QD size makes it unlikely that two QDs in the observed region of the sample will have the same fundamental exciton emission energy. However, the unique signature of a single anharmonic quantum emitter is photon antibunching, observed in photon correlation measurements where one measures the likelihood of photon detection at time $\tau$ given an initial photo-detection event at time $\tau=0[10]$.

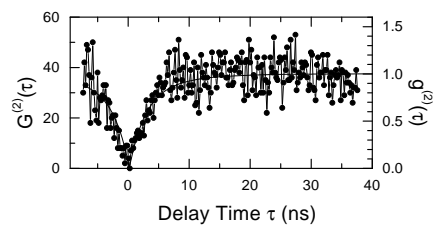

Figure 2: Photon correlation measurements carried out using single quantum dot exciton emission, under continuous-wave excitation.

Figure 2 shows the results of photon (intensity) correlation measurements carried out using a Hanbury-Brown and Twiss (HBT) set-up, under continuous-wave (cw) excitation of the QD sample. The normally ordered intensity correlation function $g^{(2)}(\tau)$ vanishes for zero-time delay between successive photo-detection events, indicating that it is impossible for the observed emitter to emit two photons simultaneously. Since $g^{(2)}(0)<0.5$, we conclude with certainty that the observed emission originates from a single QD [11].

\section{Single-photon turnstile device}

In quantum cryptography, establishment of a secret key can be achieved using single photon pulses. Recently, it has been shown that a single photon source along with high-efficiency photon counting would allow for efficient quantum computation using linear optical elements. For the latter application, it is crucial that the single-photon source has very high efficiency $(>99 \%)$ and produce indistinguishable photons that exhibit two-photon interference [14].

Photon correlation measurements carried out using a single QD under cw excitation demonstrated strong anti-correlation between successive photons, but fell short of producing a regulated source of single photons. Intensity correlation function for a single-photon turnstile device should exhibit (a) peaks at the integer multiples of its 
period (determined by the period of an external classical excitation source), indicating that photons are only emitted at well defined time-intervals; and (b) $g^{(2)}(0)=0$, indicating that none of the pulses contain more than one photon.

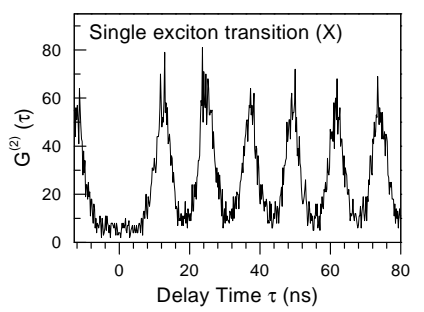

Figure 3: Photon correlation measurements carried out using single quantum dot exciton emission pumped by a mode-locked laser.

Figure 3 shows the photon correlation measurements for a single QD pumped by a mode-locked laser, where peaks at integer multiples of the pump laser period are clearly visible. The peak at $\tau=0$ however, is completely absent in the photon correlation function of the single QD fundamental exciton emission. In contrast, for the coherent mode-locked laser that pumps the QD, the $\tau=0$ peak is as strong as the other peaks [3].

\section{Cavity quantum electrodynamics using quan- tum dots}

Microdisk cavities support whispering gallery modes (WGMs) where photons are confined by total internal reflection in the disk circumference. These WGMs can have high $\mathrm{Q}$ values with relatively small mode volumes $(Q=12,000$ for a $2 \mu \mathrm{m}$ microdisk, with mode volume $V_{\text {cav }} \sim 50\left(\lambda / 2 n_{r}\right)^{3}$ ), which in turn makes them suitable for cavityQED studies despite the fact that they do not provide directional emission. WGM resonances are characterized by azimuthal $(\mathrm{M})$ and radial $(\mathrm{N})$ mode numbers, where M determines the azimuthal dependence $e^{i M}$ and $N-1$ denotes the number of nodes in the radial direction. The modes exhibit a two-fold degeneracy due to the azimuthal dependence (M, and -M). The WGMs with $N=1$ are contained within a ring of width $\lambda / 2 n_{r}$; for our microdisk structures only low radial number modes $(N=1,2,3)$ are observable since the post (with refractive index comparable to that of the disk) provides large optical losses for high radial number WGMs. We have analyzed GaAs microdisk samples containing InAs QDs in the center plane. First, a $0.5 \mu \mathrm{m} A l_{0.65} G a_{0.35} A s$ post layer was grown by MBE followed by the $200 \mathrm{~nm}$ GaAs disk layer. QDs were embedded in the center plane of the $200 \mathrm{~nm}$ disk layer. The QD density of our sample was $2 \times 10^{6} \mathrm{~cm}^{-2}$. Microdisk structures were then produced using a two-step wet etching process and contained a few QDs randomly located in the center plane. A typical microdisk sample containing a single InAs QD is shown in Figure 4. 


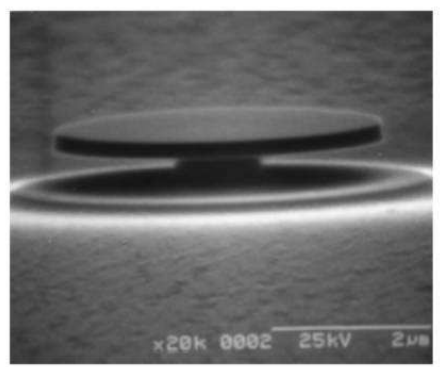

Figure 4: A $5 \mu \mathrm{m}$ diameter microdisk structure, with a single embedded InAs quantum dot.

For the single QD embedded in a microdisk cavity, we tuned the $1 \mathrm{X}$ and WGM emission lines into resonance with each other by changing the temperature of the sample. Temperature tuning relies on the relatively stronger dependence of the $1 \mathrm{X}$ emission on temperature compared to the WGM, allowing a tuning range of $\sim 3 \mathrm{meV}$ over a temperature range of $4-50 \mathrm{~K}$. The temperature dependence of the energy of the WGM is due to the change in the refractive index of GaAs with temperature, while the temperature dependence of the energy of the $1 \mathrm{X}$ transition is caused primarily by the changes in the band gap energy of InAs.

To demonstrate the Purcell effect in the weak coupling cavity-QED regime, we carried out time-correlated single photon counting (TCPC) experiments at three different temperatures as shown in Figure 5 [13]. In these experiments the pulsed laser emission and the $1 \mathrm{X}$ emission are detected by two single photon counting APDs. Output of the APD illuminated by the laser emission (1X emission) is sent to the start (stop) input of a time-to-amplitude converter whose output is stored in a multichannel analyzer (MCA). In the low excitation regime where our experiments were carried out, the biexciton emission or emission from the higher excited states were not observed. In this limit, the decay time in the coincidence histogram corresponds to the lifetime of the $1 \mathrm{X}$ transition. In Fig. 5, measured (identical) decay times of $1.8 n s$ at $4 \mathrm{~K}$ and 48 $\mathrm{K}$ show that when the $1 \mathrm{X}$ transition is not coupled to a WGM its lifetime does not depend on sample temperature, at least in this temperature range. We can therefore conclude that nonradiative recombination does not significantly effect the dynamics of the $1 \mathrm{X}$ transition for $T \leq 50 \mathrm{~K}$. When the $1 \mathrm{X}$ emission is in resonance with the WGM at $31 \mathrm{~K}$, the measured decay time is reduced to $850 \mathrm{ps}$, implying a Purcell factor of at least 2 . We note here that the electron-hole pairs in the wetting layer have average recombination times of $\sim 300 \mathrm{ps}$ or larger depending on the existence and nature of trap states. For our low density QD samples, we measured lifetimes as long as $1 n s$ on the low energy side of the wetting layer luminescence using the TCPC technique. Hence, the measured decay time in our case may be limited by the lifetime of the wetting layer states that pump the QD. For our specific WGM $(Q=8300$, $V_{\text {cav }} \sim 240\left(\lambda / 2 n_{r}\right)^{3}$, an ideal Purcell effect of $F_{P}=22$ is estimated assuming an ideal spatial match between the QD and WGM. 


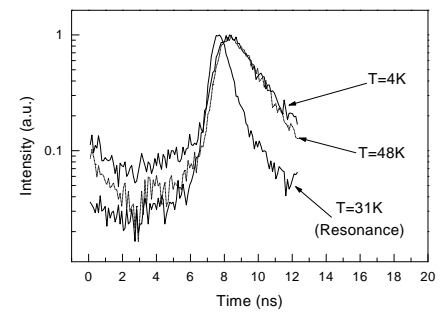

Figure 5: Time-correlated single-photon counting measurements for the fundamental exciton line of a InAs quantum dot at various temperatures.

Besides providing an enhancement in the radiative recombination rate, the Purcell effect also ensures that photons are primarily emitted into a specific cavity mode. These properties are essential for realizing fast and efficient semiconductor single photon sources, as discussed in the next Section.

\section{Outlook}

The principal factors that limit QD quantum optics in general and cavity-QED in particular, are the spatial and spectral overlap of QD resonances with high-Q cavity modes. Recent developments in ordered growth of self assembled QDs on prepatterned surfaces is likely to provide the required control over QD location . The spectral overlap, however, is more tricky due to poor control we have over QD and cavity resonance energies. The temperature tuning technique discussed in the previous section is far from optimal, since increasing the sample temperature enhances the dipole dephasing rate, potentially prohibiting the observation of strong coupling regime. Utilization of ac-Stark effect induced on the QD fundamental exciton transition by a red-detuned laser field on the other hand, could provide a dephasing-free method to tune QDs into resonance with high-Q cavity-modes. It must be said however that the uncertainty in QD energies is typically much larger than the tuning range available with either of these techniques, which in turn implies that several QDs need to be placed inside the cavity-mode to ensure that one of them could be tuned into resonance. Control of QD exciton energies during growth would certainly constitute a breakthrough for cavity-QED experiments. Techniques for tuning the cavity-mode energy independently of the QD resonance also needs to be explored.

A possible method for utilizing QDs in quantum information processing is in triggered single-photon generation. A significant fraction of early key experiments in the field of quantum information, such as quantum key distribution and quantum teleportation, have been carried out using photons and linear optical elements such as polarizers and beam splitters. However, it was generally believed that the role of linear optics in quantum information processing was limited to these specific applications, due to the difficulty in implementing conditional quantum dynamics using single photons. In contrast, recent work of Knill, Laflamme, and Milburn [14] demonstrated that efficient quantum computation is possible using linear optics (LOQC).

The principal requirement for LOQC is the presence of a single-photon source that generates indistinguishable photons. This can be achieved only if the generated pho- 
tons are Fourier-transform limited, which is in turn guaranteed if the fundamental exciton transition is broadened predominantly by spontaneous emission. Experiments on InAs QDs embedded in bulk semiconductors demonstrated that the linewidth is at most twice as large as that of radiative broadening. Enhancement of the radiative decay by the Purcell effect could then ensure that the generated photons are transform limited. A second important requirement for LOQC is the high-efficiency (> 99\%) of the single-photon source: while this is a very demanding requirement, the only way to meet it would be to use directional output from a cavity that one could obtain using a large Purcell effect. We believe that QD cavity-QED has the potential to provide such a high-efficiency transform-limited single photon source, without requiring strong-coupling regime.

\section{Acknowledgement}

The work presented here has been carried out in collaboration with A. Kiraz, P. Michler, C. Becher, B. Gayral, L. Zhang, E. Hu, W. Schoenfeld, and P. Petroff. Financial support was provided by the Packard Foundation and ARO.

\section{References}

[1] A. Zrenner, J. of Chem. Phys. 112, 7790 (2000).

[2] P. M. Petroff, A. Lorke, and A. Imamoglu, Physics Today 54, 46 (2001).

[3] P. Michler, A. Kiraz, C. Becher, W. V. Schoenfeld, P. M. Petroff, L. Zhang, E. $\mathrm{Hu}$, and A. Imamoglu, Science 290, 282 (2000)

[4] C. Santori, M. Pelton, G. S. Solomon, Y. Dale, and Y. Yamamoto, Phys. Rev. Lett. 86, 1502 (2001).

[5] Z. Yuan, B. E. Kardynal, R. M. Stevenson, A. J. Shields, C. J. Lobo, D. A. Ritchie, M. L. LEadbeater, and M. Pepper, Science 295, 102 (2002).

[6] E. Moreau, I. Robert, L. Manin, V. Thierry-Mieg, J.-M. Gerard, and I. Abram, Phys. Rev. Lett. 87, 183601 (2001).

[7] A. Kiraz, S. Flth, C. Becher, B. Gayral, W. V. Schoenfeld , P. M. Petroff, L. Zhang, E. Hu, and A. Imamoglu Phys. Rev. B 65, 161303(R) (2002).

[8] L. C. Andreani, G. Panzarini, and J.-M. Gerard, Phys. Rev. B 60, 13276 (1999).

[9] J.-M. Gerard, B. Sermage, B. Gayral, B. Legrand, E. Costard, V. Thierry-Meig, Phys. Rev. Lett. 81, 1110 (1998).

[10] R. Loudon, The Quantum Theory of Light, Oxford University Press, Oxford (1985).

[11] C. Becher, A. Kiraz, P. Michler, A. Imamoglu, W. V. Schoenfeld, P. M. Petroff, Lidong Zhang, and E. Hu, Phys. Rev. B 63, 121312(R) (2001).

[12] E. M. Purcell, Phys. Rev. 69, 681 (1946).

[13] A. Kiraz, P.Michler, C. Becher, L. Zhang, E.Hu, W.V. Schoenfeld, P.M. Petroff, A. Imamoglu, Appl. Phys. Lett. 78, 3932 (2001).

[14] E. Knill, R. Laflamme, and G. Milburn, Nature 409, 46 (2001). 